\title{
Protein intake and bone health: a systematic review and meta-analysis
}

\author{
Andrea Darling ${ }^{1}$, David Torgerson ${ }^{2}$, Joe Millward ${ }^{1}$ and Susan Lanham-New ${ }^{1}$ \\ ${ }^{1}$ University of Surrey, Guildford, UK and ${ }^{2}$ University of York, York, UK
}

The impact of dietary protein on bone mass density $(\mathrm{BMD})^{(1)}$, bone turnover ${ }^{(2)}$ and fracture risk ${ }^{(3)}$ has been widely researched. Theoretically, protein should benefit bone as a result of its influence on the anabolic hormone insulin-like growth factor 1 . However, dietary protein also increases acid load to the body, thus encouraging calciuria, which may be detrimental to bone health. There is a considerable lack of consensus as to whether protein has a beneficial or detrimental effect on bone. Despite the importance of this topic, no systematic review and meta-analysis of studies has been undertaken to date to examine the relationship between protein intake and bone health.

The aim of the present study was to conduct a systematic review and meta-analysis of the effect of dietary protein on BMD, bone turnover and fracture risk. MEDLINE (January 1966-September 2007) was searched for all cross-sectional, longitudinal and experimental studies of healthy participants. A total of twenty-one studies were included in the systematic review and six intervention studies in the meta-analysis. All chosen studies were checked by a least two researchers.

As shown in the Table, the systematic review showed a positive correlation between total dietary protein and BMD in adults, with the variation in low BMD that was attributable to protein intake being approximately $2-4 \%$. In the longitudinal studies the mean relative risk for any fracture in the highest quartile of total protein intake was 0.7. Some intervention studies showed an increase in markers of bone formation and a decrease in markers of bone resorption with protein supplementation and some found a benefit on BMD. No significant effect was found in the meta-analysis for lumbar spine BMD $(P=0.17, \mathrm{Z} 1.36$, weighted mean difference $0.05(95 \% \mathrm{CI}-0.02,0.12)$ ). The only significant effect found in the meta-analysis was for the effect of soybean protein on bone-specific alkaline phosphatase (BSAP), favouring the control group $(P=0.04, \mathrm{Z} 2.08$, weighted mean difference 0.03 (95\% CI $0.00,0.07)$ ).

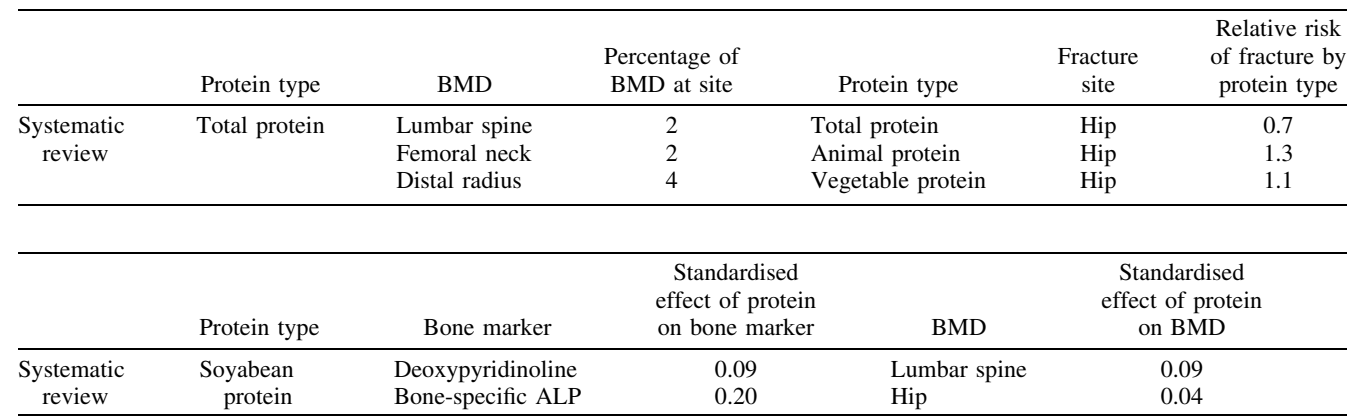

These effects suggest that dietary protein may have a beneficial effect on BMD. However, the long-term effect on fracture risk is unclear, and intervention studies have not supported the findings of the cross-sectional and longitudinal studies. However, this disparity may be a result of the poor quality and heterogeneity of the intervention studies, or confounding in the cross-sectional studies. Further research in this area is urgently required.

1. Vatanparast H, Bailey DA, Baxter-Jones AG \& Whiting SJ (2007) J Nutr 137, 2674-2679.

2. Ince B, Anderson EJ \& Neer RM (2004) J Clin Endocrinol Metab 89, 3801-3807.

3. Wengreen HJ, Munger RG, West NA, Cutler DR, Corcoran CD, Zhang J \& Sassano NE (2004) J Bone Miner Res 19, 537-545. 